Radiologe 2020 60:839-849

https://doi.org/10.1007/s00117-020-00684-4

Online publiziert: 29. Mai 2020

(C) Der/die Autor(en) 2020

Sabine Dettmer ${ }^{1}$ - Sabine Schneidewind ${ }^{2}$. Volkhard Fischer ${ }^{3}$ Katja Derlin ${ }^{1}$. Nils Schneider ${ }^{4} \cdot$ Frank Wacker $^{1} \cdot$ Kambiz Afshar $^{4}$

${ }^{1}$ Institut für Diagnostische und Interventionelle Radiologie, Medizinische Hochschule Hannover, Hannover, Deutschland

${ }^{2}$ Klinik für Gastroenterologie, Hepatologie und Endokrinologie, Medizinische Hochschule Hannover, Hannover, Deutschland

${ }^{3}$ Studiendekanat, Bereich Evaluation \& Kapazität, Medizinische Hochschule Hannover, Hannover, Deutschland

${ }^{4}$ Institut für Allgemeinmedizin, Medizinische Hochschule Hannover, Hannover, Deutschland

\title{
Training zur strukturierten Befundung von Radiographien des Thorax mit OSCE-Prüfung
}

\section{Ergebnisse einer Machbarkeitsstudie und Follow-up-Befragung}

\section{Hintergrund und Fragestellung}

Mit dem Masterplan Medizinstudium 2020 erfährt die medizinische Ausbildung einen Wandel hin zu einer zunehmend kompetenzbasierten Ausbildung mit der schwerpunktmäßigen Vermittlung von Kompetenzen, Fertigkeiten und Haltungen [2, 19]. $\mathrm{Zu}$ den wesentlichen Reformzielen zählt die Vermittlung von Kompetenzen sowie die konsequente Integration von grundlagenwissenschaftlichen und klinischen Inhalten über den gesamten Verlauf des Studiums mit frühzeitigem Patientenund Praxisbezug [19]. Mit dem Nationalen Kompetenzbasierten Lernzielkatalog Medizin (NKLM) liegen seit dem Jahr 2015 Empfehlungen für die kompetenzbasierte Ausrichtung der medizinischen Ausbildung vor $[4,13]$.

Eine wichtige Grundkompetenz ärztlichen Handelns ist die strukturierte Befundung von Radiographien des Thorax. Entsprechende Lernziele sind z. B. in den Kapiteln 15.4.4-6 des NKLM 1.0 formuliert [13]. Klinisch tätige Ärztinnen und Ärzte sollten befähigt sein, eigenständig und ohne Konsultation eines Radiologen eine strukturierte Befundung vornehmen zu können. Von besonderer Bedeutung ist dies z.B. in Notaufnah- men ohne durchgehende Präsenz von Radiologen und auch im niedergelassenen Bereich, wo Radiographien mit entsprechender Fachkunde auch von anderen Fachdisziplinen angefertigt und beurteilt werden. Es ist daher sinnvoll, bereits frühzeitig in der medizinischen Ausbildung entsprechende Fähigkeiten und Fertigkeiten zu vermitteln, die als Grundlage für den Kompetenzerwerb dienen können.

Obwohl die Mehrzahl der Lehrstuhlinhaber für das Fach Radiologie an den medizinischen Fakultäten in Deutschland einen integrativen und interdisziplinären Aufbau der radiologischen Lehre befürworten, stellt sich die Ausgestaltung heterogen dar. Gründe sind insbesondere der hohe organisatorische und zeitliche Aufwand [3]. Eine Einbindung der Radiologie früh im Studium scheint insbesondere deswegen sinnvoll, da viele Studierende bereits im Rahmen von Famulaturen nach Ende des 2. Studienjahres Umgang mit Radiographien des Thorax haben.

Um dies umzusetzen, wurden national und international in den letzten Jahren bereits innovative Lehrkonzepte mit praktischem Training z.B. als sog. Blended-learning-Einheiten erfolgreich implementiert $[6,11]$, in der klassische Lehrmethoden in Form von Präsenzveranstaltungen und E-Learning miteinander kombiniert werden. Neben problemorientiertem Unterricht in Kleingruppen werden auch zunehmend interaktive fallbasierte Trainings an Computern eine wichtige Rolle spielen. Entsprechend wurden auch Prüfungsformate angepasst und die Inhalte zunehmend in mündlich-praktischen Prüfungen, z.B. in Form einer „objektive structured clinical examination“ (OSCE), geprüft $[5,18]$.

Ziel dieser Studie war es, ein neu entwickeltes curriculares Training für die strukturierte Befundung von Radiographien des Thorax mit anschließendem OSCE im Modul „Diagnostische Methoden“'des 2. Studienjahres an der Medizinischen Hochschule Hannover zu evaluieren. Mit einer schriftlichen Befragung der Studierenden sollte untersucht werden, inwieweit sich die Studierenden nach diesem Training in der Befundung von Radiographien des Thorax sicher und den Erwartungen der betreuenden ärztlichen Kollegen im Rahmen einer Famulatur gewachsen fühlen. 


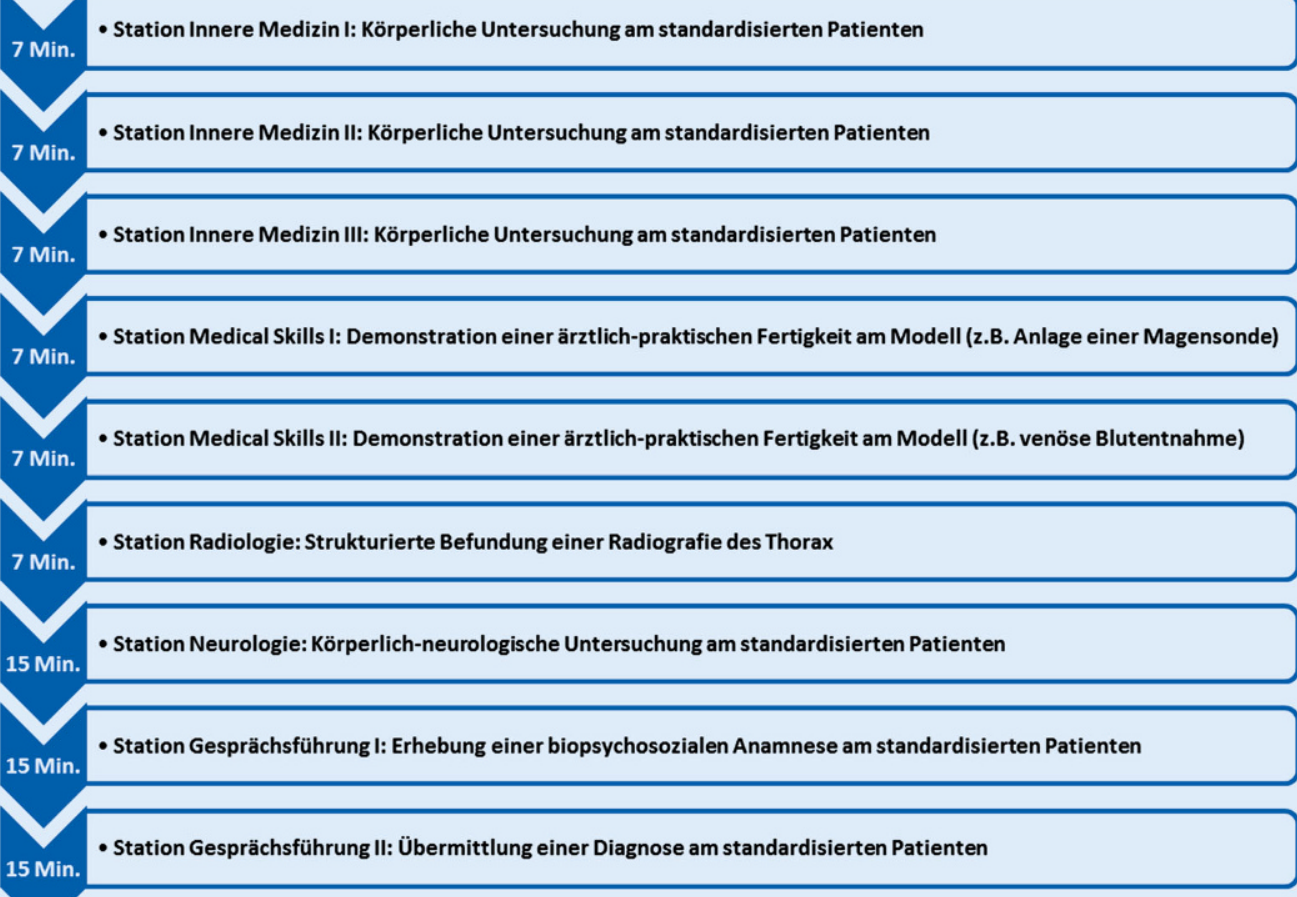

Abb. $1 \triangleleft$ Aufbau des OSCE(,objective structured clinical examination")-Parcours an der Medizinischen Hochschule Hannover mit Angaben zu Thema, Inhalt und Dauer der neun Stationen. Zwischen den Stationen besteht eine Wechselzeit von einer Minute (ParcoursGesamtdauer: $95 \mathrm{~min}$ )

\section{Studiendesign und Unter- suchungsmethoden}

\section{Studiendesign}

Es handelt sich um eine Singlezenterstudie mit prospektivem Design zur Evaluation eines neu implementierten Trainings zur strukturierten Befundung von Radiographien des Thorax im 2. Studienjahr an der Medizinischen Hochschule Hannover mit anschließender OSCE-Prüfung. Hierfür wurde eine schriftliche Befragung von Studierenden des 4. Studienjahres in zwei aufeinander folgenden Jahrgängen mit und ohne Training durchgeführt. Einschlusskriterien waren alle Studierende, die im Studienjahr 2016/17 im 4. Studienjahr waren und alle Studierenden, die im Studienjahr 2017/18 im 4. Studienjahr waren und an dem ersten OSCE mit Radiologie teilgenommen hatten (jeweils 269 angeschriebene Studierende). Die vorliegende Studie wurde von der Ethikkommission der Medizinischen Hochschule Hannover begutachtet und genehmigt (Nr. 3323-2016).

\section{Implementierung des neuen Trainings zur strukturierten Befundung von Radiographien des Thorax}

An der Medizinischen Hochschule Hannover wurde zum Studienjahr 2015/16 die strukturierte Befundung von Radiographien des Thorax in das Modul „Diagnostische Methoden" des 2. Studienjahres neu implementiert. Die Grundlagen zur systematischen Befundung konventioneller Radiographien des Thorax in 2 Ebenen wurden im Rahmen von insgesamt vier neuen Vorlesungseinheiten (Dauer jeweils eine Unterrichtsstunde) und videoassistierten Schulungen vermittelt. Bei Letzteren handelt es sich um kommentierte Lehrfilme, die zunächst die strukturierte Befundung eines Normalbefundes anhand eines anatomischen Templates demonstrieren und dann in 10 Lehrfilmen typische Befunde bei häufigen Pathologien behandeln. Die Lehrfilme beinhalten auch Fragen an die Studierenden, die sie zunächst eigenständig bearbeiten sollen und deren Lösung im weiteren Verlauf des Films gegeben und erläutert wird. Die Filme können von den Studierenden jederzeit online abgerufen werden. Neben der anatomischen Orientierung in der Radiographie des Thorax und der strukturierten Befundung stand das selbstständige Erkennen grundlegender und für den späteren Berufsalltag relevanter Erkrankungen, wie z.B. Pneumothorax, Pneumonie, Pleuraerguss und Lymphom, im Vordergrund. Die Anwesenheit der Studierenden in den Vorlesungen und die Nutzung der Lehrvideos waren fakultativ und wurden nicht überprüft.

\section{Radiologie-Station im OSCE}

Die vermittelten Inhalte wurden erstmals in einer interdisziplinären OSCE im Studienjahr 2015/16 geprüft. Die OSCEPrüfung im 2. Studienjahr umfasst pro Prüfling 9 Stationen à 7 oder $15 \mathrm{~min}$ Dauer, mit einer Gesamtprüfungsdauer von $95 \mathrm{~min}$. Eine Übersicht zum Ablauf des OSCE ist in $\bullet$ Abb. 1 gegeben. Es handelt sich um einen kumulativen OSCE mit einer Bestehensgrenze von 180 von maximal 300 Punkten (60\%). Die Prüfung erfolgt in einem abgedunkelten Raum, und es wird ein radiologischer Befundungsmonitor verwendet. Die radiologische Station dauert 7 Minuten und 
Radiologe 2020 - 60:839-849 https://doi.org/10.1007/s00117-020-00684-4

(c) Der/die Autor(en) 2020

S. Dettmer · S. Schneidewind · V. Fischer · K. Derlin · N. Schneider · F. Wacker · K. Afshar

Training zur strukturierten Befundung von Radiographien des Thorax mit OSCE-Prüfung. Ergebnisse einer Machbarkeitsstudie und Follow-up-Befragung

\section{Zusammenfassung}

Hintergrund. Die strukturierte Befundung von Radiographien des Thorax stellt eine wichtige radiologische Grundkompetenz dar und wird mit der Umsetzung des nationalen kompetenzbasierten Lernzielkatalogs Medizin (NKLM) in der studentischen Lehre gefordert. Ziel der Arbeit. Ziel war die Evaluierung, inwieweit Studierende des 2. Studienjahres durch ein neues curriculares Training zur Befundung von Radiographien des Thorax mit anschließender "objective structured clinical examination" (OSCE) an Sicherheit gewinnen und wie gut sie sich den Erwartungen der betreuenden ärztlichen Kollegen während der Famulatur gewachsen fühlen.

Methoden. Das Training umfasste 4 Vorlesungen und 10 kommentierte Lehrfilme zur
Befundung konventioneller Radiographien. Die Studierenden wurden 2 Jahre später befragt, wie häufig und in welcher Form sie in Famulaturen Umgang mit Radiographien hatten und wie sicher sie sich im Umgang in verschiedenen Teilbereichen fühlten. Die Studierenden mit Training wurden zusätzlich gefragt, inwiefern Vorlesungen und Lehrvideos hilfreich waren. Die Ergebnisse der Studierenden des letzten Jahrgangs ohne und des ersten Jahrgangs mit Training und OSCE wurden mittels Mann-Whitney-U-Test für unabhängige Stichproben verglichen. Ergebnisse. Die Häufigkeit des Umgangs mit Radiographien war bei Studierenden mit und ohne Training vergleichbar hoch. Die Studierenden mit Training und OSCE fühlten sich im weiteren Studium sicherer und besser vorbereitet als die Studierenden ohne Training (je nach Teilbereich $p<0,001$ bis $p=0,148$ ). Sie bewerteten die Lehrfilme als hilfreicher als die Vorlesungen (Mittelwert: 2,85 $\pm 0,76$ vs. $3,41 \pm 0,68)$.

Diskussion. Mit einem frühzeitigen Training kann die Sicherheit der Studierenden im Umgang mit Radiographien des Thorax nachhaltig gesteigert werden.

Schlüsselwörter

Medizinische Ausbildung · Röntgen .

Famulatur $\cdot$ Lehrfilm $\cdot$ Selbsteinschätzung

\section{Structured chest X-ray imaging training with OSCE examination. Results of a feasibility study and follow-up survey}

\section{Abstract}

Background. The structured diagnosis of chest radiographs is an important basic competence in radiology and will be required in the student teaching with the implementation of the National Competency-Based Learning Catalog Medicine (NKLM) in Germany. Aim. The aim was to evaluate the extent to which second-year students gain confidence in the diagnosis of chest radiographs through a new curricular training for the diagnosis of chest radiographs with subsequent objective structured clinical examination (OSCE) and how confident they feel about meeting the expectations of the supervising medical colleagues during the clinical clerkship.
Methods. The training included four lectures and ten commented instructional films on the diagnosis of radiographs. Two years later, the students were asked how often and in what form they had contact with chest radiographs in their clinical clerkship. They were also asked how confident they felt in dealing in different areas. The students with training were also asked to what extent the lectures and the instructional films were helpful. The results of students in the last year without and the first year with training and OSCE were compared using the Mann-Whitney $\mathrm{U}$ test for independent samples.

Results. The frequency of dealing with chest radiographs was comparably high among students with and without training. The students with training and OSCE felt more confident and better prepared in dealing with chest radiographs than the students without training (varying between $p<0.001$ and $p=0.148$ ). They rated the educational films as more helpful than the lectures (mean $2.85 \pm 0.76$ vs. $3.41 \pm 0.68$ on a four-point scale).

Discussion. With early training, students' confidence in dealing with chest radiographs can be sustainably increased.

Keywords

Medical education - Thoracic X-ray · Clinical clerkship · Instructional film · Self-assessment es können maximal 25 Punkte erreicht werden. Inhaltlich umfasst sie die strukturierte Befundung von Radiographien des Thorax anhand von verschiedenen Fallbeispielen wie z. B. Pneumothorax, Pneumonie, Pleuraerguss und Lymphom, von denen die Studierenden jeweils einen Fall bearbeiteten. Ähnliche aber nicht identische Fälle wurden zuvor in den Vorlesungen und Lehrvideos behandelt. Die Kategorien der Checkliste umfassen dabei unabhängig vom Thema die Aufgabenbereiche Aufnahmetechnik und Qualität (z. B. Kriterien zur Beurteilung der Aufnahmetechnik; 4 Punkte), anatomische Orientierung und strukturierte Befundung (z.B. Durchführung einer strukturierten Befundung, Abfragen relevanter anatomischer Strukturen; 14 Punkte) sowie Erkennen und Deuten von Pathologien (z.B. richtige Diagnose stellen, Röntgenzeichen der verschiedenen Pathologien nennen und zeigen; 7 Punkte).

\section{Befragung}

Evaluiert wurde das Vorhaben durch eine anonymisierte webbasierte Befragung von Studierenden, die das Training noch nicht (keine Vorlesungen und keine Lehrvideos zum praktischen Erlernen einer strukturierten Radiographie des Thorax, keine Radiologie im OSCE), und solchen, die das Training erstmalig durchlaufen haben. Die Umfrage erfolgte in Zusammenarbeit mit dem Evaluationsbü- 
Tab. 1 Fragen aus der Umfrage zur Häufigkeit des Umgangs mit Radiographien während der Famulatur, zu den Fähigkeiten und der Sicherheit der Studierenden bei der Befundung von Radiografien des Thorax sowie zu den Vorlesungen, Lehrvideos und der OSCE zum Erlernen der strukturierten Befundung von Radiografien des Thorax

Fragen zur Häufigkeit des Umgangs mit Radiographien während der Famulatur

Ich habe einen schriftlichen Befund zu einer konventionellen Thoraxaufnahme gelesen, aber nicht das Bild dazu gesehen

Ich habe eine konventionelle Thoraxaufnahme z. B. während der Röntgendemo, der Visite oder im Rahmen einer Besprechung gezeigt bekommen

Mir wurde eine konventionelle Thoraxaufnahme z. B. von einem ärztlichen Kollegen persönlich erklärt

Ich habe eine konventionelle Thoraxaufnahme z. B. auf Station oder in der Notaufnahme eigenständig betrachtet und den schriftlichen Befund versucht nachzuvollziehen

Ich habe eine konventionelle Thoraxaufnahme z. B. auf Station oder in der Notaufnahme eigenständig betrachtet und eine eigene Befundung vorgenommen

Fragen zu den Fähigkeiten und der Sicherheit der Studierenden bei der Befundung von Radiographien des Thorax Ich habe eine feste Struktur, mit der ich an die Befundung einer konventionellen Thoraxaufnahme herangehe Ich fühle mich sicher bei der Betrachtung einer konventionellen Thoraxaufnahme

Ich setzte mich mit einer konventionellen Thoraxaufnahme auseinander und versuche, sie zu verstehen

Ich kann mich in einer konventionellen Thoraxaufnahme anatomisch orientieren

Ich kann in einer konventionellen Thoraxaufnahme eine Pathologie erkennen

Ich kann in einer konventionellen Thoraxaufnahme eine Pathologie deuten und benennen

Ich habe das Gefühl, dass ich den Erwartungen der ärztlichen Kollegen zur Befundung einer konventionellen Thoraxaufnahme gewachsen bin

Ich habe das Gefühl, dass ich, was die Befundung eines Röntgenthorax angeht, für meine zukünftige Tätigkeit gut gerüstet bin

Fragen zu den Vorlesungen, Lehrvideos und der OSCE zum Erlernen der strukturierten Befundung von Radiographien des Thorax

Die Vorlesungen waren hilfreich für das Erlernen der strukturierten Befundung des Röntgenthorax

Die Lehrvideos waren hilfreich für das Erlernen der strukturierten Befundung des Röntgenthorax

Durch die Radiologie-Station in der OSCE-Prüfung habe ich mich intensiver mit dem Thema auseinandergesetzt als es ohne die Prüfung der Fall gewesen wäre

Ich habe nach der OSCE-Prüfung häufiger und bewusster konventionelle Thoraxuntersuchungen betrachtet

Ich fühle mich durch die Vorbereitung auf die OSCE-Prüfung Radiologie gut für die Befundung von konventionellen Thoraxaufnahmen vorbereitet ro der Medizinischen Hochschule Hannover jeweils im 4 . Studienjahr in zwei aufeinanderfolgenden Jahren, dem Studienjahr 2016/17 (269 Studierende, letzter Jahrgang ohne Training und OSCE, Kontrollgruppe) und dem Studienjahr 2017/18 (269 Studierende, erster Jahrgang mit Training und OSCE, Interventionsgruppe). Über das Studiendekanat wurden alle Studierenden des jeweiligen Jahrgangs per E-Mail angeschrieben und zur Teilnahme an der anonymisierten Umfrage eingeladen. Die Umfrage erfolgte webbasiert mit Hilfe der Evaluationssoftware EvaSys 7.0 (Electric Paper Evaluationssysteme GmbH, Lüneburg, Deutschland). pothesengeleitet ein teilstandardisierter Fragebogen mit 14 Frageitems entwickelt. Es wurde auf einer vierstufigen Antwortskala (1=nie; $2=$ selten, $1-3 /$
Im interdisziplinären Team wurde hy-
Woche; 3 = häufig, 4-10/Woche; $4=$ sehr häufig, > 10/Woche) gefragt, ob, wie häufig und in welcher Form seit Abschluss der OSCE-Prüfung Umgang mit Radiographien des Thorax bestand: Lesen eines schriftlichen Befundes, Teilnahme an Demonstrationen in Röntgenbesprechungen, Radiographie persönlich erklärt bekommen, Radiographie selber betrachtet und/oder selber befundet. Die Studierenden wurden weiterhin auf einer vierstufigen Antwortskala $(1=$ trifft nicht zu; $2=$ trifft eher nicht zu; $3=$ trifft eher zu; $4=$ trifft voll $\mathrm{zu}$ ) gefragt, wie strukturiert sie bei der Befundung einer konventionellen Thoraxaufnahme vorgehen, wie sicher sie sich bei der anatomischen Orientierung (Teilbereich 2 des OSCE) sowie beim Erkennen bzw. Deuten von Pathologien (Teilbereich 3 des OSCE) fühlen, und ob sie sich ausreichend auf ihre ärztliche Tätigkeit

\author{
Antwortskala: \\ $1=$ nie \\ $2=$ selten ( $1-3 /$ Woche) \\ 3 = häufig (4-10/Woche) \\ $4=$ sehr häufig (>10/Woche)
}

\section{Antwortskala:}

$1=$ trifft nicht $z u$

$2=$ trifft eher nicht $z u$

$3=$ trifft eher $z u$

$4=$ trifft voll zu vorbereitet fühlen. Die einzelnen Fragen sind $\bullet$ Tab. 1 zu entnehmen.

Die Studierenden, die das Training durchlaufen hatten, wurden zusätzlich gefragt, inwieweit die Vorlesungen und die Lehrvideos hilfreich zum Erlernen der strukturierten Befundung von Radiographien des Thorax waren (vierstufige Antwortskala: $1=$ trifft nicht $\mathrm{zu}$ bis $4=$ trifft voll $\mathrm{zu}$ ) und wie häufig sie die Lehrvideos vor und nach dem OSCE angesehen haben. Da der Effekt des Trainings in der klinischen Praxis evaluiert werden sollte, wurde auch abgefragt, ob bereits eine Famulatur absolviert wurde. Soziodemographische Angaben der Studierenden, wie z. B. Alter, Geschlecht und berufliche Vorerfahrungen, wurden aus datenschutzrechtlichen Gründen nicht erhoben. 
Tab. 2 Umfrageergebnisse der 192 Studierenden zur Häufigkeit des Umgangs mit Radiographien während ihrer Famulatur: Gruppenvergleich zwischen Studierenden mit/ohne Training und OSCE mittels Mann-Whitney-U-Test. Es wurde eine vierstufige Antwortskala verwendet $(1=$ nie; $2=$ selten 1-3/Woche; 3 = häufig, 4-10/Woche; 4 = sehr häufig, $>10 /$ Woche)

\begin{tabular}{|c|c|c|c|c|c|c|c|}
\hline & \multicolumn{2}{|c|}{ Gesamt $(N=192)$} & \multicolumn{2}{|c|}{$\begin{array}{l}\text { Kontrollgruppe ( } n=93 \text {, } \\
\text { kein Training) }\end{array}$} & \multicolumn{2}{|c|}{$\begin{array}{l}\text { Interventionsgruppe } \\
(n=99, \text { mit Training) }\end{array}$} & \multirow[t]{2}{*}{$p$-Wert } \\
\hline & $\begin{array}{l}\text { Mittelwert } \\
\text { (SD) }\end{array}$ & $\begin{array}{l}\text { Median } \\
\text { (IQR) }\end{array}$ & $\begin{array}{l}\text { Mittelwert } \\
\text { (SD) }\end{array}$ & $\begin{array}{l}\text { Median } \\
\text { (IQR) }\end{array}$ & $\begin{array}{l}\text { Mittelwert } \\
\text { (SD) }\end{array}$ & $\begin{array}{l}\text { Median } \\
\text { (IQR) }\end{array}$ & \\
\hline $\begin{array}{l}\text { Ich habe einen schriftlichen Befund zu einer konven- } \\
\text { tionellen Thoraxaufnahme gelesen, aber nicht das } \\
\text { Bild dazu gesehen }\end{array}$ & $2,08(0,77)$ & $2(2-3)$ & $2,10(0,79)$ & $2(2-3)$ & $2,06(0,76)$ & $2(2-2)$ & 0,634 \\
\hline $\begin{array}{l}\text { Ich habe eine konventionelle Thoraxaufnahme z. B. } \\
\text { während der Röntgendemo, der Visite oder im Rah- } \\
\text { men einer Besprechung gezeigt bekommen }\end{array}$ & $2,84(0,99)$ & $3(2-4)$ & $2,93(0,92)$ & $3(2-4)$ & $2,76(1,04)$ & $3(2-4)$ & 0,231 \\
\hline $\begin{array}{l}\text { Mir wurde eine konventionelle Thoraxaufnahme z.B. } \\
\text { von einem ärztlichen Kollegen persönlich erklärt }\end{array}$ & $\begin{array}{l}2,18 \\
(0,84)\end{array}$ & $\begin{array}{l}2 \\
(2-3)\end{array}$ & $\begin{array}{l}2,13 \\
(0,79)\end{array}$ & $\begin{array}{l}2 \\
(2-3)\end{array}$ & $\begin{array}{l}2,23 \\
(0,90)\end{array}$ & $\begin{array}{l}2 \\
(2-3)\end{array}$ & 0,492 \\
\hline $\begin{array}{l}\text { Ich habe eine konventionelle Thoraxaufnahme z. B. } \\
\text { auf Station oder in der Notaufnahme eigenständig } \\
\text { betrachtet und den schriftlichen Befund versucht } \\
\text { nachzuvollziehen }\end{array}$ & $2,05(0,91)$ & $2(1-3)$ & $1,98(0,89)$ & $2(1-2)$ & $2,11(0,93)$ & $2(1-3)$ & 0,286 \\
\hline $\begin{array}{l}\text { Ich habe eine konventionelle Thoraxaufnahme z.B. } \\
\text { auf Station oder in der Notaufnahme eigenständig } \\
\text { betrachtet und eine eigene Befundung vorgenom- } \\
\text { men }\end{array}$ & $1,38(0,70)$ & $1(1-2)$ & $1,31(0,65)$ & $1(1-1)$ & $1,45(0,74)$ & $1(1-2)$ & 0,101 \\
\hline SD Standardabweichung, IQR Interquartilsabstand & & & & & & & \\
\hline
\end{tabular}

\section{Statistische Auswertung}

Die statistische Auswertung erfolgte mit der Statistiksoftware IBM SPSS Statistics Version 24.0 (IBM Corporation, USA). Für die Darstellung der Ergebnisse wurden deskriptive Statistiken (Mittelwert, Median, Prozentwerte) verwendet. Vergleiche zwischen den Gruppen erfolgten mittels Mann-Whitney-U-Test für unabhängige Stichproben. Das gewählte Signifikanzniveau für die Ablehnung der Nullhypothese betrug für alle durchgeführten Tests $a<0,05$.

\section{Ergebnisse}

\section{Studienpopulation}

Insgesamt 262 Studierende des 4 . Studienjahres haben an der schriftlichen Befragung teilgenommen, davon $145 \mathrm{Stu}$ dierende in der Gruppe mit Training und OSCE (Interventionsgruppe) und 117 in der Gruppe ohne Training und OSCE (Kontrollgruppe). Bei jeweils 269 angeschriebenen Studierenden ergab sich eine Teilnahmequote von $55 \%$ in der Interventionsgruppe verglichen mit $43 \%$ in der Kontrollgruppe. 36 (14\%) Studierende hatten zum Zeitpunkt der Umfrage noch keine Famulatur absolviert. 225 Stu- dierende (86\%) hatten bereits mindestens eine Famulatur abgeschlossen, davon 101 Studierende (45\%) eine, 81 Studierende (36\%) zwei und 43 Studierende $(19 \%)$ mehr als zwei Famulaturen. Davon hatten 192 Studierende (85\%) in der Famulatur Umgang mit Radiographien, hierunter 93 Studierende $(79 \%)$ in der Kontrollgruppe (ohne Training und OSCE) und 99 Studierende (68\%) in der Interventionsgruppe (mit Training und OSCE).

Beim OSCE schnitten die Studierenden beider Studienjahre vergleichbar ab. Im Studienjahr 2014/15 erreichten die Studierenden $(N=259)$ im Mittel 253 Punkte (Standardabweichung: 21 Punkte, Median: 259 Punkte). Im Studienjahr 2015/16 erreichten die Studierenden $(N=267)$ im Mittel 248 Punkte (Standardabweichung: 22 Punkte, Median: 252 Punkte).

\section{Radiographien in der Famulatur}

Es gab keinen signifikanten Unterschied zwischen den Studierenden mit und ohne Training für die Befundung konventioneller Radiographien des Thorax hinsichtlich der Häufigkeit des Umgangs mit Radiographien des Thorax während ihrer Famulatur. Auch in der weiteren Spezi- fizierung z. B. Lesen eines Befundes, Demonstration einer Radiographie, Erklärung einer Radiographie, eigenständige Betrachtung einer Radiographie und eigene Befunderstellung zeigten sich keine signifikanten Unterschiede zwischen den beiden Studierendengruppen ( $\boldsymbol{0}$ Tab. 2; - Abb. 2).

\section{Fähigkeiten und Sicherheit im Umgang mit Radiographien des Thorax}

In der Selbsteinschätzung der Fähigkeiten und der Sicherheit in der Befundung von Radiographien des Thorax unterschieden sich die Studierenden mit und ohne Training signifikant ( $\bullet$ Tab. 3; - Abb. 3). Studierende mit Training gaben signifikant häufiger an, eine feste Struktur bei der Befundung von Radiographien des Thorax zu haben (Mittelwert: 2,46 vs. 1,$92 ; p<0,001)$ und sich sicherer bei der Betrachtung zu fühlen (Mittelwert: 1,99 vs. 1,$56 ; p<0,001$ ). Ebenso sahen sich Studierende mit vorangegangenem Training eher in der Lage, sich in der Radiographie anatomisch zu orientieren (Mittelwert: 3,24 vs. 3,05; $p=0,022)$, eine Pathologie zu erkennen (Mittelwert: 2,78 vs. 2,$61 ; p=0,017$ ) und diese $\mathrm{zu}$ deuten (Mittelwert: 2,33 vs. 


\section{Originalien}

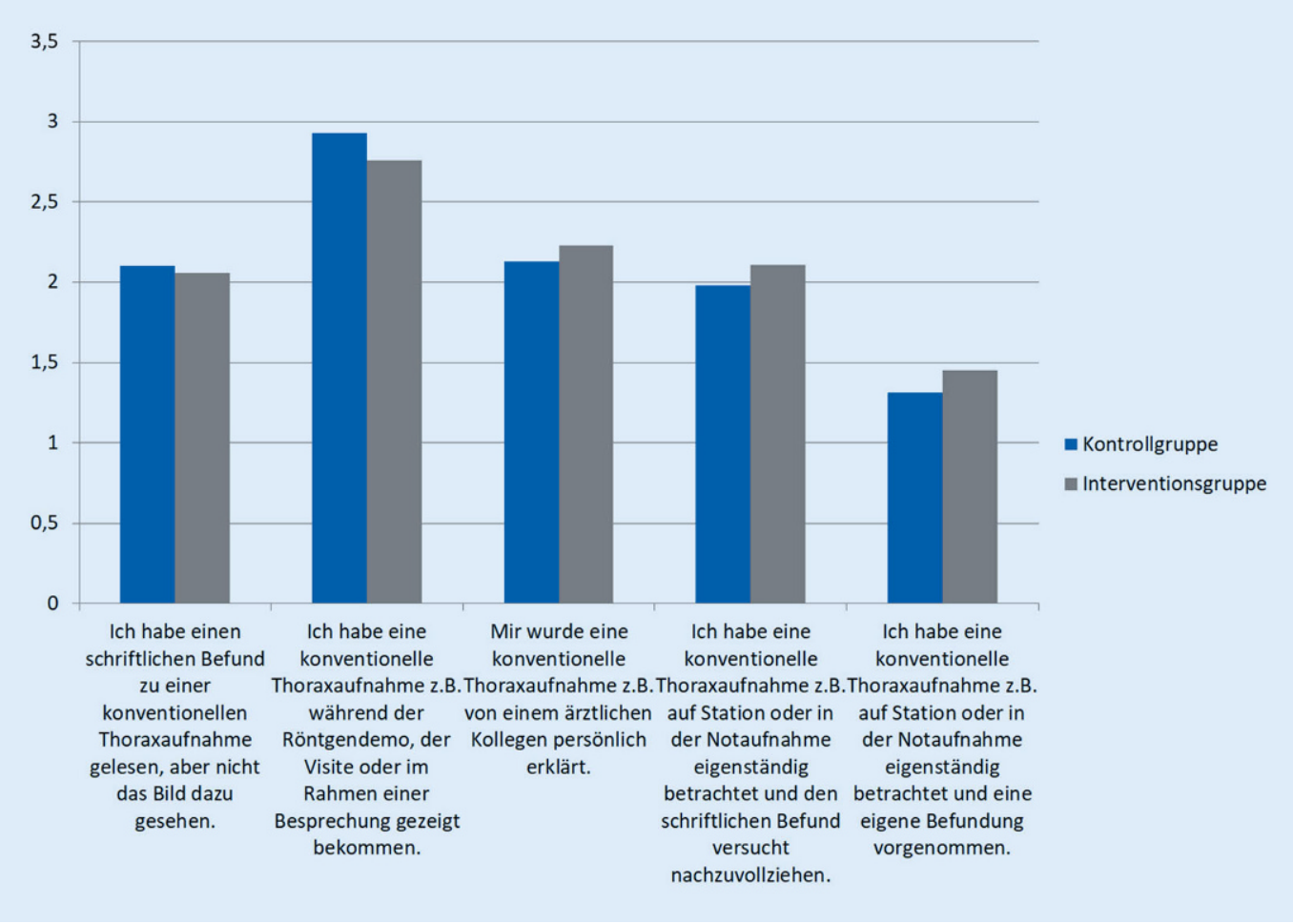

Abb. $2 \triangleleft$ Boxplot der studentischen Umfrageergebnisse zur Häufigkeit im Umgang mit Radiographien des Thorax während der Famulatur, unterteilt in Interventionsgruppe und Kontrollgruppe

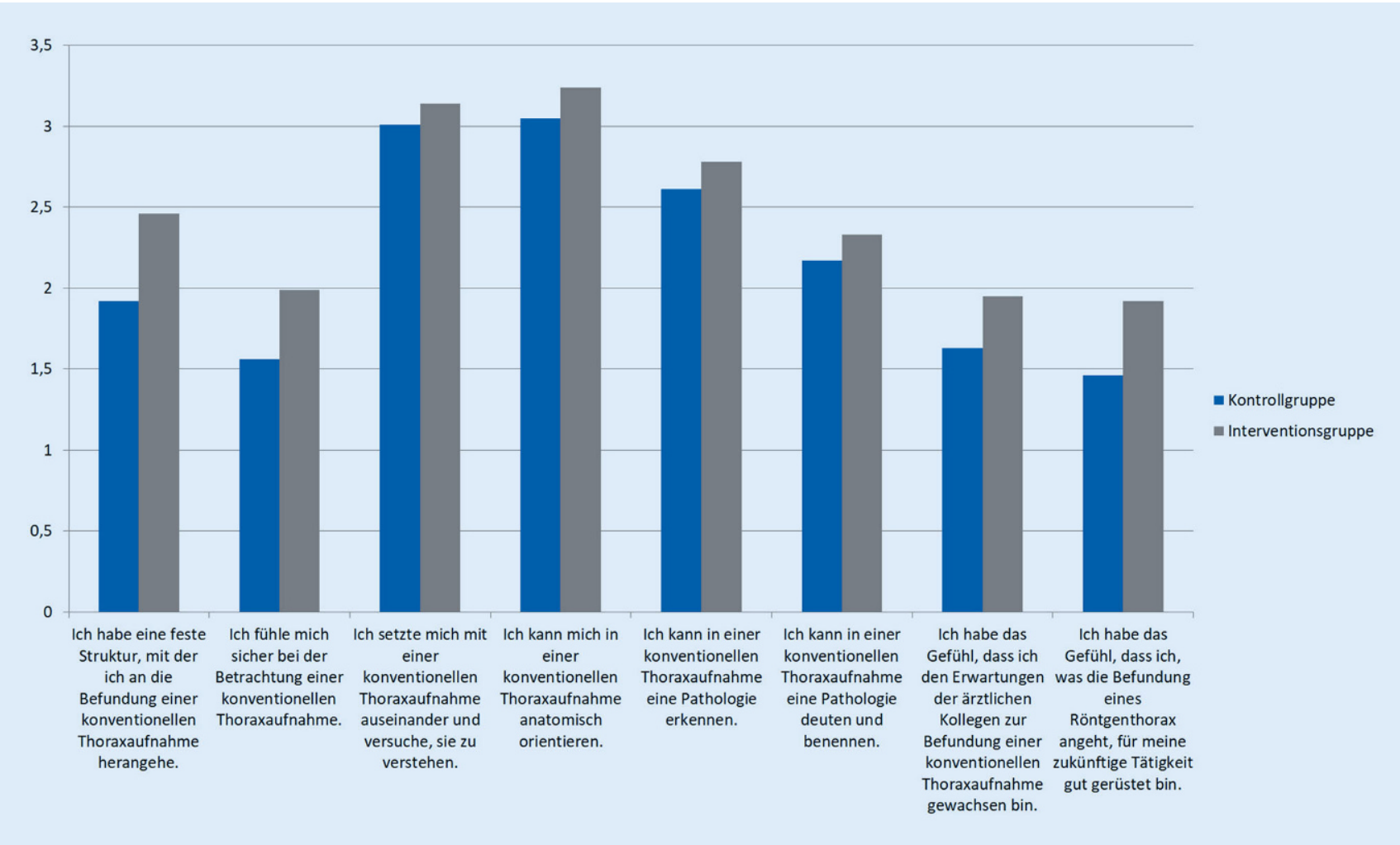

Abb. $3 \Delta$ Boxplot der studentischen Umfrageergebnisse zur Sicherheit in der Befundung von Radiographien des Thorax, unterteilt in Interventionsgruppe und Kontrollgruppe 
Tab. 3 Umfrageergebnisse aller 192 Studierenden zu ihren Fähigkeiten und ihrer Sicherheit bei der Befundung von Radiographien des Thorax: Gruppenvergleich zwischen Studierenden mit/ohne Training und OSCE mittels Mann-Whitney-U-Test. Es wurde eine vierstufige Antwortskala verwendet ( 1 = trifft nicht zu; 2 = trifft eher nicht zu; 3 = trifft eher zu; 4 = trifft voll zu)

\begin{tabular}{|c|c|c|c|c|c|c|c|}
\hline & \multicolumn{2}{|c|}{ Gesamt $(N=192)$} & \multicolumn{2}{|c|}{$\begin{array}{l}\text { Kontrollgruppe ( } n=93 \text {, } \\
\text { kein Training) }\end{array}$} & \multicolumn{2}{|c|}{$\begin{array}{l}\text { Interventionsgruppe } \\
\text { ( } n=99, \text { mit Training) }\end{array}$} & \multirow[t]{2}{*}{$p$-Wert } \\
\hline & $\begin{array}{l}\text { Mittelwert } \\
\text { (SD) }\end{array}$ & $\begin{array}{l}\text { Median } \\
\text { (IQR) }\end{array}$ & $\begin{array}{l}\text { Mittelwert } \\
\text { (SD) }\end{array}$ & $\begin{array}{l}\text { Median } \\
\text { (IQR) }\end{array}$ & $\begin{array}{l}\text { Mittelwert } \\
\text { (SD) }\end{array}$ & $\begin{array}{l}\text { Median } \\
\text { (IQR) }\end{array}$ & \\
\hline $\begin{array}{l}\text { Ich habe eine feste Struktur, mit der ich an die } \\
\text { Befundung einer konventionellen Thoraxauf- } \\
\text { nahme herangehe }\end{array}$ & $2,22(0,90)$ & $2(1-3)$ & $1,92(0,87)$ & $2(1-3)$ & $2,46(0,85)$ & $3(2-3)$ & $<0,001$ \\
\hline $\begin{array}{l}\text { Ich fühle mich sicher bei der Betrachtung einer } \\
\text { konventionellen Thoraxaufnahme }\end{array}$ & $1,80(0,72)$ & $2(1-2)$ & $1,56(0,60)$ & $2(1-2)$ & $1,99(0,75)$ & $2(1-2)$ & $<0,001$ \\
\hline $\begin{array}{l}\text { Ich setzte mich mit einer konventionellen Tho- } \\
\text { raxaufnahme auseinander und versuche, sie zu } \\
\text { verstehen }\end{array}$ & $3,08(0,72)$ & $3(3-4)$ & $3,01(0,74)$ & $3(3-3)$ & $3,14(0,70)$ & $3(3-4)$ & 0,148 \\
\hline $\begin{array}{l}\text { Ich kann mich in einer konventionellen Thorax- } \\
\text { aufnahme anatomisch orientieren }\end{array}$ & $3,16(0,67)$ & $3(3-4)$ & $3,05(0,70)$ & $3(3-3)$ & $3,24(0,64)$ & $3(3-4)$ & 0,022 \\
\hline $\begin{array}{l}\text { Ich kann in einer konventionellen Thoraxaufnah- } \\
\text { me eine Pathologie erkennen }\end{array}$ & $2,70(0,61)$ & $3(2-3)$ & $2,61(0,59)$ & $3(2-3)$ & $2,78(0,61)$ & $3(2-3)$ & 0,017 \\
\hline $\begin{array}{l}\text { Ich kann in einer konventionellen Thoraxaufnah- } \\
\text { me eine Pathologie deuten und benennen }\end{array}$ & $2,26(0,63)$ & $2(2-3)$ & $2,17(0,59)$ & $2(2-3)$ & $2,33(0,65)$ & $2(2-3)$ & 0,020 \\
\hline $\begin{array}{l}\text { Ich habe das Gefühl, dass ich den Erwartungen } \\
\text { der ärztlichen Kollegen zur Befundung einer } \\
\text { konventionellen Thoraxaufnahme gewachsen } \\
\text { bin }\end{array}$ & $1,81(0,72)$ & $2(1-2)$ & $1,63(0,67)$ & $2(1-2)$ & $1,95(0,73)$ & $2(1-2)$ & $<0,001$ \\
\hline $\begin{array}{l}\text { Ich habe das Gefühl, dass ich, was die Befun- } \\
\text { dung eines Röntgenthorax angeht, für meine } \\
\text { zukünftige Tätigkeit gut gerüstet bin }\end{array}$ & $1,72(0,73)$ & $2(1-2)$ & $1,46(0,59)$ & $1(1-2)$ & $1,92(0,76)$ & $2(1-2)$ & $<0,001$ \\
\hline SD Standardabweichung, IQR Interquartilsabstand & & & & & & & \\
\hline
\end{tabular}

Tab. 4 Umfrageergebnisse der 145 Studierenden mit Training zu der Frage, wie hilfreich Vorlesungen, Lehrvideos und der OSCE zum Erlernen der strukturierten Befundung von Radiographien des Thorax waren auf einer vierstufigen Antwortskala ( $1=$ trifft nicht zu; $2=$ trifft eher nicht zu; $3=$ trifft eher zu; $4=$ trifft voll zu)

\begin{tabular}{|c|c|c|}
\hline & Mittelwert (SD) & Median (IQR) \\
\hline Die Vorlesungen waren hilfreich für das Erlernen der strukturierten Befundung des Röntgenthorax & $2,85(0,76)$ & $3(2-3)$ \\
\hline Die Lehrvideos waren hilfreich für das Erlernen der strukturierten Befundung des Röntgenthorax & $3,41(0,68)$ & $3(3-4)$ \\
\hline $\begin{array}{l}\text { Durch die Radiologie-Station in der OSCE-Prüfung habe ich mich intensiver mit dem Thema auseinanderge- } \\
\text { setzt, als es ohne die Prüfung der Fall gewesen wäre }\end{array}$ & $3,23(0,86)$ & $3(3-4)$ \\
\hline Ich habe nach der OSCE-Prüfung häufiger und bewusster konventionelle Thoraxuntersuchungen betrachtet & $2,64(0,88)$ & $3(2-3)$ \\
\hline $\begin{array}{l}\text { Ich fühle mich durch die Vorbereitung auf die OSCE-Prüfung Radiologie gut für die Befundung von konven- } \\
\text { tionellen Thoraxaufnahmen vorbereitet }\end{array}$ & $2,27(0,73)$ & $2(2-3)$ \\
\hline SD Standardabweichung, IQR Interquartilsabstand & & \\
\hline
\end{tabular}

2,17; $p=0,020)$. Studierende mit Training fühlten sich besser auf ihre spätere ärztliche Tätigkeit vorbereitet (Mittelwert: 1,92 vs. 1,$46 ; p<0,001)$ und den Erwartungen der ärztlichen Kollegen zur Befundung einer Radiographie des Thorax eher gewachsen (Mittelwert: $1,95$ vs. 1,$63 ; p<0,001)$. Die Bereitschaft, sich mit einer Radiographie des Thorax intensiv auseinanderzusetzen, war bei Studierenden mit und ohne Training vergleichbar hoch (Mittelwert: 3,14 vs. $3,01 ; p=0,148)$.

\section{Trainingsinhalte}

Bei der Befragung der Studierenden mit Training zeigte sich (•Tab.4), dass insbesondere das Training mit den Lehrvideos (Mittelwert: 3,41) für das Erlernen der strukturierten Befundung von Radiographien des Thorax als hilfreich erachtet wurde und dies deutlich besser eingeschätzt wurde als die Vorbereitung durch die Vorlesungen (Mittelwert: $2,85)$. Die meisten Studierenden stimmten darin überein, dass sie sich durch die
Radiologie-Station in der OSCE-Prüfung intensiver mit dem Thema auseinandergesetzt haben, als es ohne die Prüfung der Fall gewesen wäre (Mittelwert: 3,23). Der Großteil der Studierenden war der Ansicht, sich nach der OSCE-Prüfung häufiger und bewusster mit Radiographien des Thorax beschäftigt zu haben (Mittelwert: 2,64). Dennoch fühlten sich viele Studierende durch die Vorbereitung auf die Radiologie-Station in der OSCEPrüfung noch unzureichend auf die Be- 
fundung von Radiographien des Thorax vorbereitet (Mittelwert: 2,27).

\section{Diskussion}

Wir konnten mit unserer Studie zeigen, dass unser neu implementiertes curriculares Röntgen-Thorax-Training mit anschließendem OSCE den Studierenden in ihrer Famulatur mehr Sicherheit bei der strukturierten Befundung von Radiographien des Thorax gibt und sie eher den Eindruck haben, den Erwartungen der betreuenden Ärzte und den Anforderungen bei ihrer späteren ärztlichen Tätigkeit gewachsen zu sein.

\section{Sicherheit im Umgang mit Radiographien}

Wir konnten zeigen, dass sich die Studierenden mit dem Training und der OSCEStation Radiologie im weiteren Verlauf ihres Studiums sicherer in der Befundung von Radiographien des Thorax fühlten als die Studierenden ohne Training. Das Gefühl der Sicherheit betraf alle Teilbereiche: von dem strukturierten Vorgehen und der anatomischen Orientierung in der Radiographie über das Erkennen der Pathologie hin zur richtigen Interpretation. Auch waren Studierende mit Training häufiger der Überzeugung, den Erwartungen der ärztlichen Kollegen in dieser Hinsicht gewachsen und für ihre zukünftige Tätigkeit ausreichend gerüstet zu sein. Es ist bekannt, dass studentische Selbsteinschätzungen bestimmten Störgrößen unterliegen können und Studierende dazu neigen, ihre Fertigkeiten und Kompetenzen zu überschätzen [1, 7, 8]. Diese Effekte müssten jedoch in beiden Gruppen vergleichbar sein, der Unterschied bezüglich der Sicherheit zwischen den Studierenden mit und ohne Training war jedoch signifikant. Zudem gibt es auch Hinweise darauf, dass es zwischen der studentischen Selbstsicherheit und Selbsteinschätzung einen positiven Zusammenhang gibt [10].

Weiterhin deuten die Ergebnisse darauf hin, dass der Aspekt der eigenen Sicherheit bei der Befundung nachhaltig zu sein scheint, da die Angaben in der Befragung 2 Jahre nach dem Training und nach den ersten praktischen Erfahrungen in Famulaturen gemacht wurden. Eine objektive Überprüfung, z. B. mittels erneuter OSCE-Prüfung, steht noch aus. Es ist denkbar, dass die erworbenen Fertigkeiten und die damit verbundene Sicherheit der Studierenden in Zusammenhang stehen. Die Häufigkeit des Umgangs mit Radiographien während der Famulatur war bei Studierenden mit und ohne Training hingegen vergleichbar. Offensichtlich wird weder von den Studierenden mit Training aktiv der Umgang mit Radiographien gesucht noch von den Studierenden ohne Training vermieden. Die erlernten Fertigkeiten haben somit keinen Einfluss auf die Häufigkeit des Umgangs mit Radiographien, sondern eher die Gegebenheiten bei der Famulatur.

\section{Lehrformate des Trainings}

Bei der Vermittlung der radiologischen Trainingsinhalte haben die Studierenden den Lehrfilmen einen größeren Stellenwert zugesprochen als den Vorlesungen. Dies könnte dadurch erklärt werden, dass durch das Training verschiedene Lerntypen angesprochen werden (auditiv, visuell und konstruktiv) und sich die Studierenden aktiver, wiederholt und im eigenen Lerntempo mit den Lerninhalten auseinandersetzen konnten. Folienbasierte Vorlesungen haben sich für die Vermittlung vieler Lehrinhalte bewährt, stoßen aber an ihre Grenzen, wenn Studierende aktiv beteiligt und wenn in einem praktischen Training Fähigkeiten und Fertigkeiten vermittelt werden sollen. Durch die zunehmende Digitalisierung bieten sich sehr viel mehr Möglichkeiten, die Lehre interaktiv zu gestalten [8-11]. Die Radiologie eignet sich hierzu in besonderer Weise und durch die überwiegend bildbasierten Lehrinhalte besser als die meisten anderen Fachdisziplinen. Die positive Rückmeldung zu den Lehrvideos zeigt, dass eine hohe Akzeptanz dieses Lehrformats bei den Studierenden besteht. Dennoch zeigte unsere Befragung, dass sich die Studierenden mit Training besser als die ohne Training aber immer noch nicht ausreichend auf die Befundung konventioneller Thoraxaufnahmen vorbereitet fühlen. Ein ergänzendes interaktives webbasiertes Lernmodul mit Fällen zur selbstständigen Be- fundung mit begleitendem Seminar wird derzeit bei uns implementiert und soll diesen Bedarf decken. Die Kombination aus Blended-learning-Einheiten und fallbasiertem Unterricht scheint das selbstgesteuerte Lernen und das Lernergebnis positiv zu beeinflussen $[11,12]$. Dabei ist aber zu beachten, dass für beide Lehrmittel zwar ein hoher Erstellungsaufwand notwendig ist, sie letztlich aber als Teil des Eigenstudiums nicht angemessen auf die Lehrleistung der Dozierenden angerechnet werden können. Damit wird ihre nachhaltige Einbindung in die curriculare Lehre unnötig durch die rechtlichen Rahmenbedingungen gebremst.

\section{Radiologie-Station im OSCE}

Die Entwicklung von Checklisten mit Ankerkriterien für die Prüfung der im Training vermittelten radiologischen Inhalte erfolgte in einem interdisziplinären Team mit Expertise in Radiologie, Innerer Medizin, Allgemeinmedizin und Medizindidaktik. Hierbei hat sich die Aufteilung in die drei zentralen Themenbereiche Aufnahmetechnik und Qualität (4 Punkte), anatomische Orientierung und strukturierte Befundung (14 Punkte) sowie Erkennen und Deuten von Pathologien (7 Punkte) als sinnvoll erwiesen, sowohl für die Entwicklung der Prüfungsmatrizen und der Ankerkriterien als auch für die transparente und zwischen den verschiedenen Matrizen vergleichbare Wichtung der erwarteten Prüfungsinhalte. Die Studierenden hatten aufgrund der gewählten Punkteverteilung zudem die Möglichkeit, durch die ersten beiden Aufgabenbereiche $72 \%$ der maximalen Punktzahl an der Radiologie-Station zu erreichen. Die Entscheidung für die Integration von Inhalten zur Beurteilung und ersten Einordnung von Pathologien wurde jedoch bewusst getroffen, um einerseits bereits in diesem frühen Stadium der medizinischen Ausbildung Befunde in den Kontext der verschiedenen Krankheitsbilder einordnen und pathophysiologisch deuten zu können. Andererseits sollte die Graduierung der erbrachten Leistung differenzierter ermöglicht werden. Das Wissen darüber, dass Inhalte des Trainings auch Inhalt einer eigenen 
Prüfungsstation sein werden, hat die Auseinandersetzung mit den radiologischen Inhalten und deren Vertiefung im Sinne des Konstruktes „assessment for learning“ verstärkt [17].

\section{Curriculum Radiologie und Bezug zum NKLM}

Im NKLM wird eine kompetenzbasierte Lehre gefordert; die strukturierte Befundung von Radiographien des Thorax gehört zweifelsohne dazu. Unser RöntgenThorax-Training ist eine Möglichkeit, dies praktisch umzusetzen. Viele Lehrende fürchten bei der Umsetzung des NKLM einen höheren personellen und zeitlichen Aufwand. Die Kombination aus Vorlesung und digitalen Inhalten in Form von Lehrvideos hält, wenn diese erst einmal erstellt sind, diesen Aufwand im Rahmen. Ähnliche Formate, die in diesem Zusammenhang hilfreich sein könnten, sind beispielsweise webbasierte Trainings $[9,15,16]$. Ergänzend zum dargestellten Training wurde neben einem interaktiven webbasierten Lernmodul ein einstündiges Seminar in Kleingruppen im Modul „Diagnostische Methoden“ des 2. Studienjahres eingeführt, bei dem die Studierenden Gelegenheit haben, offene Fragen zu den Fällen zu diskutieren und unter Supervision die Befundung zu trainieren. Hinsichtlich des Zeitpunktes für das Röntgen-Thorax-Training hat sich die frühe Implementierung im 2. Studienjahr als günstig erwiesen. Aus Sicht der Studierenden und Lehrverantwortlichen scheint die Vermittlung grundlegender Fähigkeiten und Fertigkeiten bereits früh im Studium sinnvoll, da diese Grundlage für den Kompetenzerwerb ist, und dazu haben die Studierendenden in den Famulaturen nur bei frühzeitiger Lehre ausreichend Zeit.

\section{Limitationen}

Eine Limitation der Studie ist die fehlende Leistungskontrolle in der Kontrollgruppe. Diese konnte nicht erfolgen, zum einen war eine zusätzliche Prüfung aller Studierenden des Jahrgangs ähnlich dem OSCE gegenüber den Studierenden nicht vertretbar und zum anderen hätte eine solche Prüfung bereits eine für die Kontrollgruppe nicht gewollte Intervention bedeutet.

Des Weiteren bleibt in der Befragung unklar, welche weiteren Faktoren die Sicherheit im Umgang mit Radiographien des Thorax noch beeinflusst haben könnten, insbesondere in der Studierendengruppe ohne Training. Die Items des Evaluationsfragebogens sind jedoch sehr stark auf das Training und die OSCE-Prüfung fokussiert, sodass zumindest die getroffenen Aussagen als hinreichend verwertbar angesehen werden können. Die Frage, wie nachhaltig die Sicherheit bei der Befundung auch über das 4. Studienjahr hinaus ist, soll in einem nächsten Projekt mit einer vergleichenden Untersuchung bei Studierenden im Praktischen Jahr mit und ohne Training erfolgen.

\section{Fazit für die Praxis}

\section{- Ein frühzeitiges und strukturiertes Röntgen-Thorax-Training steigert die Sicherheit Studierender im Um- gang mit Radiographien des Thorax nachhaltig. \\ - Mit dem Röntgen-Thorax-Training kann eine kompetenzbasierte Aus- gestaltung der medizinischen Aus- bildung mit adäquatem Aufwand umgesetzt werden. \\ - Die Kombination aus Vorlesungen und digitalen Medien in Form von kommentierten Lehrfilmen sind für das Röntgen-Thorax-Training besonders geeignet und werden von Studierenden akzeptiert.}

\section{Korrespondenzadresse}

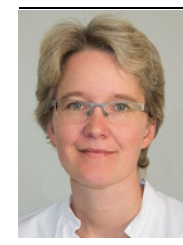

Sabine Dettmer

Institut für Diagnostische und Interventionelle Radiologie, Medizinische Hochschule Hannover Carl-Neuberg-Str. 1, 30625 Hannover, Deutschland Dettmer.Sabine@ mh-hannover.de

Danksagung. Wir möchten insbesondere den Studierenden, die an der Umfrage teilgenommen haben, für ihre Mitarbeit danken. Des Weiteren gilt unser Dank Herrn Klaas Brandt vom Studiendekanat der Medizinischen Hochschule Hannover für die Unterstützung bei der Nutzung von EvaSys sowie Frau Heike Steinlandt vom Institut für Diagnostische und Interventionelle Radiologie für die Hilfe bei der Datenerhebung.

Funding. Open Access funding provided by Projekt DEAL.

\section{Einhaltung ethischer Richtlinien}

Interessenkonflikt. S. Dettmer, S. Schneidewind, V. Fischer, K. Derlin, N. Schneider, F. Wacker und K. Afshar geben an, dass kein Interessenkonflikt besteht.

Für diesen Beitrag wurden von den Autoren keine Studien an Menschen oder Tieren durchgeführt. Für die aufgeführten Studien gelten die jeweils dort angegebenen ethischen Richtlinien. Von allen an der Befragung Beteiligten liegt eine schriftliche Einverständniserklärung vor. Die vorliegende Studie wurde von der Ethikkommission der Medizinischen Hochschule Hannover begutachtet und genehmigt (Nr. 3323-2016).

Open Access. Dieser Artikel wird unter der Creative Commons Namensnennung 4.0 International Lizenz veröffentlicht, welche die Nutzung, Vervielfältigung, Bearbeitung, Verbreitung und Wiedergabe in jeglichem Medium und Format erlaubt, sofern Sie den/die ursprünglichen Autor(en) und die Quelle ordnungsgemäß nennen, einen Link zur Creative Commons Lizenz beifügen und angeben, ob Änderungen vorgenommen wurden.

Die in diesem Artikel enthaltenen Bilder und sonstiges Drittmaterial unterliegen ebenfalls der genannten Creative Commons Lizenz, sofern sich aus der Abbildungslegende nichts anderes ergibt. Sofern das betreffende Material nicht unter der genannten Creative Commons Lizenz steht und die betreffende Handlung nicht nach gesetzlichen Vorschriften erlaubt ist, ist für die oben aufgeführten Weiterverwendungen des Materials die Einwilligung des jeweiligen Rechteinhabers einzuholen.

Weitere Details zur Lizenz entnehmen Sie bitte der Lizenzinformation auf http://creativecommons.org/ licenses/by/4.0/deed.de.

\section{Literatur}

1. Blanch-Hartigan D (2011) Medical students' selfassessment of performance: results from three meta-analyses. Patient Educ Couns 84:3-9. https:// doi.org/10.1016/j.pec.2010.06.037

2. Bundesministerium für Bildung und Forschung (2017) Masterplan Medizinstudium 2020

3. Dettmer S, Weidemann J, Fischer V et al (2015) Integrative teaching in radiology - a survey. Rofo 187(4):260-268. https://doi.org/10.1055/s-00341399193

4. Fischer MR, BauerD, Mohn K, NKLM-Projektgruppe (2015) Finally finished! National Competence Based Catalogues of Learning Objectives for Undergraduate Medical Education (NKLM) and Dental Education (NKLZ) ready for trial. GMS Z Med Ausbild 32(3):Doc35. https://doi.org/10. 3205/zma000977 
5. Harden RM, Stevenson M, Downie WW, Wilson GM (1975) Assessment of clinical competence using objective structured examination. Br Med J 1:447-451

6. Howlett D, Vincent T, Watson G et al (2011) Blending online techniques with traditional face to face teaching methods to deliver final year undergraduate radiology learning content. Eur J Radiol 78:334-341. https://doi.org/10.1016/j. ejrad.2009.07.028

7. Jünger J, Schellberg D, Nikendei C (2006) Student's self-assessment of clinical competence and objective clinical performance in OSCE evaluation. GMSZMed Ausbild 23(3):Doc51

8. Katowa-Mukwato P, Banda S (2016) Self-perceived versus objectively measured competence in performing clinical practical procedures by final year medical students. Int J Med Educ 7:122-129. https://doi.org/10.5116/ijme.5709.2a7e

9. Koestner W, Otten W, Kaireit T et al (2017) Competency-based teaching in radiology-implementation and evaluation of interactive workstation-based learning to apply NKLM-based content. Rofo 189:1076-1085. https://doi.org/10. 1055/s-0043-117888

10. Leopold SS, Morgan HD, Kadel NJ et al (2005) Impact of educational intervention on confidence and competence in the performance of a simple surgical task. J Bone Joint Surg Am 87:1031-1037. https://doi.org/10.2106/JBJS.D.02434

11. Mahnken AH, Baumann M, Meister M et al (2011) Blended learning in radiology: Is self-determined learning really more effective? Eur J Radiol 78:384-387. https://doi.org/10.1016/j.ejrad.2010. 12.059

12. Maleck M, Fischer MR, Kammer B et al (2001) Do computers teach better? A media comparison study for case-based teaching in radiology. Radiographics 21:1025-1032. https://doi.org/10. 1148/radiographics.21.4.g01jl091025

13. Medizinischer Fakultätentag (2015) Nationaler Kompetenzbasierter Lernzielkatalog Medizin (NKLM). http://www.nklm.de/files/nklm_final 2015-07-03.pdf;. Zugegriffen:30. Jan. 2017

14. Rojas CA, Jawad H, Chung JH (2012) The new era of radiology teaching files. AJR Am J Roentgenol 198:773-776. https://doi.org/10.2214/AJR.11. 7409

15. Strickland CD, Lowry PA, Petersen BD, Jesse MK (2015) Introduction of a virtual workstation into radiology medical student education. AJR Am J Roentgenol 204:W289-W292. https://doi.org/10. 2214/AJR.14.13180

16. Tam MDBS, Hart AR, Williams SM et al (2010) Evaluation of a computer program ('disect') to consolidate anatomy knowledge: a randomisedcontrolled trial. Med Teach 32:e138-e142. https:// doi.org/10.3109/01421590903144110

17. Thistlethwaite J (2006) More thoughts on "assessment drives learning". Med Educ 40:1149-1150. https://doi.org/10.1111/j.1365-2929.2006.02638. $x$

18. Verhoeven BH, Hamers JG, Scherpbier AJ et al (2000) The effect on reliability of adding a separate written assessment component to an objective structured clinical examination. Med Educ 34:525-529

19. Wissenschaftsrat (2018) Neustrukturierung des Medizinstudiums und Änderung der Approbationsordnung für Ärzte. Empfehlungen der Expertenkommission zum Masterplan Medizinstudium 2020

\section{Künstliche Intelligenz für die Notfallmedizin}

Neues Verbundprojekt entwickelt eine Software, die CT-Aufnahmen automatisch nach verdächtigen Zeichen für bestimmte Befunde absucht und auf dringliche Probleme hinweist.

Ziel des Projekts „Künstliche Intelligenz (KI) für radiologische Bildgebung in der Notfall- und Intensivmedizin" (KI-RAD) ist es, einen intelligenten Röntgenassistenten zu entwickeln, der dabei hilft, wichtige Informationen aus Röntgen- und CTBildern zu filtern, die entscheidend für die weitere Versorgung von Patientinnen und Patienten sind. Hierfür wurden drei kritische Anwendungsbereiche ausgewählt: Schlaganfall, Knochenverletzungen und Röntgenthorax.

\section{Intelligenter Röntgenassistent soll dringliche Probleme erkennen} Bei Verdacht auf einen Schlaganfall zählt z.B. jede Minute. Wichtig ist vor allem, schnell zu differenzieren, ob ein verschlossenes Blutgefäß die Symptome verursacht oder eine Hirnblutung.

Bei Verletzungen von Knochen soll das KI-gestützte Analysesystem zum einen zwischen frischen Frakturen infolge eines Unfalls und alten Brüchen, beispielsweise als Folge von Osteoporose, unterscheiden. Zum anderen gilt es, instabile Brüche zu erkennen, die eine besondere Vorsicht im Umgang mit Betroffenen erfordern. Bei Wirbelkörperfrakturen besteht die Gefahr, dass das Rückenmark verletzt wird und Lähmungen auftreten. Bei dem Anwendungsbereich Röntgenthorax geht es darum, Probleme zu erkennen, die einer schnellen Behandlung bedürfen, zum Beispiel bei Atemnot auf Grund eines Pneumothorax oder auch die richtige Platzierung von Kathetern zu überprüfen.

\section{Beispielbilder für automatisierte} Diagnoseverfahren

Grundlage für die Entwicklung des intelligenten Röntgenassistenten sind CT-Bilder, die von spezialisierten Radiologinnen und Radiologen interpretiert und kategorisiert wurden. Das KI-System lernt aus diesen Beispielbildern, erkennt Muster und Gesetzmäßigkeiten. Zusätzlich können auch Informationen von anderen Bildgebungsmethoden, etwa der MRT, genutzt werden, um das System zu trainieren.

Potenzielle Anwendungsgebiete für ein KI- gestütztes Analyseverfahren gibt es viele. Zum Beispiel könnten kleinere Kliniken, denen die entsprechende radiologische Expertise fehlt, es nutzen. Auch ein Einsatz zu Trainingszwecken sei vorstellbar.

\section{Über KI-RAD}

Das auf drei Jahre angelegte Projekt "Künstliche Intelligenz (KI) für radiologische Bildgebung in der Notfall- und Intensivmedizin" (KI-RAD) ist ein Anwendungsprojekt innerhalb von „KI-Space für intelligente $\mathrm{Ge}$ sundheitssysteme" (KI-SIGS). Es wird mit 1,5 Millionen Euro vom Bundesministerium für Wirtschaft und Energie gefördert. Ziel ist die Entwicklung und Bereitstellung von Kl-gestützten Analyseverfahren zur Optimierung von Workflow und Befundungsqualität für Bildgebung in der Notfall- und Intensivmedizin, speziell in den Bereichen Schlaganfall, Skelettrauma und Intensiv/Röntgenthorax. Das Projekt startete im April 2020 und endet voraussichtlich im März 2023. Neben der CAU als Koordinatorin sind auch die Universität zu Lübeck, das Universitätsklinikum HamburgEppendorf (UKE), das Universitätsklinikum Schleswig-Holstein (UKSH) sowie die Unternehmen Philips, Hamburg, und mbits, Heidelberg, beteiligt.

\section{Weitere Informationen:}

Molecular Imaging North Competence Center (MOIN CC), www.moincc.de/

KI-SIGS, ki-sigs.de/

Quelle: Christian-Albrechts-Universität zu Kiel 
Hier steht eine Anzeige.

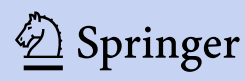

